www.jmscr.igmpublication.org

Index Copernicus Value: 79.54

ISSN (e)-2347-176x ISSN (p) 2455-0450

crossref DOI: https://dx.doi.org/10.18535/jmscr/v7i6.152

Journal Of Medical Science And Clinical Research

IGM Publication

An Official Publication of IGM Publication

\title{
Evaluation of Results of Intercondylar Fracture of Humerus in Adults Treated by Various Surgical Modalities
}

\author{
Authors \\ Dr P.V.V.S.N. Reddy ${ }^{1}$, Dr L.Prasanna Kumar ${ }^{2 *}$ \\ ${ }^{1}$ Dept. of Orthopaedics, Government Medical College \& General Hospital, Srikakulam, A.P \\ ${ }^{2}$ Dept. of Orthopaedics, ACSR Medical College \& General Hospital, Nellore, A.P \\ *Corresponding Author \\ Dr L.Prasanna Kumar \\ Dept. of Orthopaedics, ACSR Medical College \& General Hospital, Nellore, A.P, India
}

\begin{abstract}
The intercondylar fractures of humerus were treated conservatively because of lack of experience of individual surgeon and rarity of these fractures. Conservative treatment of intercondylar fractures of humerus resulted in loss of elbow movement and permanent disability. Any treatment which requires prolonged immobilization risks loss of elbow function from fibrosis or ankylosis of the joint. Due to the above problems and the recent advances which made understanding of the joint mechanics and alignment better, the need of restoration has been understood. Later, to restore the joint, many surgeons tried closed manipulation, skeletal traction and percutaneous restoration of fragments but could not achieve good results. In the recent years, with fabrication of newer implants and with better surgical exposures, results of patients with intercondylar fractures of humerus have improved a lot. In spite of all these it is well known that anyone who has operated upon these badly broken and comminuted fractures of the lower end of humerus must have been impressed by the extreme difficulty of fixing the fragments in their proper position 4. The present study is to evaluate the results of intercondylar fractures of distal humerus treated and in management with various surgical modalities. 20 cases of intercondylar fractures of humerus have been done by open reduction and internal fixation, 10 cases had excellent results, 5 had good, 3 had fair and 2 had poor results in our study. In summary, the concept of open reduction and internal fixation of intercondylar fractures of the humerus with dual plates is very valuable, in restoring articular surface and early rehabilitation decreasing morbidity, resulting good results.
\end{abstract}

\section{Introduction}

Intercondylar fractures represent one of the most complicated and challenging fractures in the upper extremity. Intercondylar fractures of the humerus in adults are difficult fractures to treat because of significant comminution. The results of managing these fractures non-operatively are limited by failure to get anatomical reduction and early mobilization, which often results in painful stiff elbow or pseudarthrosis. Hence an operative management with anatomical reduction of the fragments becomes the treatment of choice for these fractures ${ }^{1}$. The complex three-dimensional geometry of the distal humerus poses a considerable challenge to reconstruction ${ }^{2}$. 
The distal humerus fractures are rare fractures constituting $2 \%^{3}$ of all body fractures, most of them being intercondylar. Medial and lateral condyles are usually separate fragments displaced in $\mathrm{T}$ or $\mathrm{Y}$ configuration and both unconnected from the humeral shaft and rotated in axial plane. The forearm musculature originating on the condyles tends to produce rotational redisplacement even when closed reduction is achieved. The goal of treatment is to re establish the articular congruity and alignment and early post operative mobilization. In most cases open reduction with stable rigid internal fixation is required to fulfil the above goal. The treatment of severely comminuted fractures of the elbow has been a subject of controversy since long. Recommendations for treatment have ranged widely from essentially no treatment to operative reduction and extensive internal fixation. In some of these fractures particularly those with intra articular comminution, anatomical restoration of the articular surface cannot be adequately achieved or maintained through manipulative reduction alone.

Many orthopaedic surgeons stress on preserving the architecture of any joint for its normal restoration of function. The recent trend has been immediate open reduction, stable and rigid internal fixation and early post operative mobilization. The anatomic complexity of distal humerus makes surgical reconstruction difficult. The fabrication of newer implants however, has increased the reliability of the operative stabilization, while placing additional demands on the surgeon expertise. Injuries of the elbow lead to chronic pain and permanent restriction of motion, limiting the use of the hand in most activities. Positioning of hand for grip and apprehension is dominated by freedom of motion at the elbow. Basic daily activities from eating to personal hygiene require a wide range of positions and movements at the elbow in flexion, extension and forearm rotation. Any restricted motion of the neck, shoulder or wrist magnifies impairment of the elbow. More complex tasks at the work place or recreation require even greater functional demands. Recent advances in surgical techniques and equipment designed to make possible rigid osteosynthesis of smaller intra articular fragments now permit early post operative rehabilitation and achieve improved results.

An attempt has been made in this dissertation to evaluate the results of intercondylar fractures of humerus treated by various surgical modalities. Patients selected for this operation were of different ages and genders, admitted and treated in NRI general hospital, Chinakakani during the years 2015-2017. The classification criteria used was AO classification. Most of them were AO type 13.C and a few were AO type 13.B.

\section{Materials and Methods}

It is a prospective study of 20 consecutive cases of intercondylar fractures of humerus (AO Type 13.B and 13.C, 15 male and 5 females age ranging from 18 to 60 ) treated by open reduction and internal fixation over two years, from July 2015 to September 2017 was conducted at NRI MEDICAL COLLEGE AND GENERAL HOSPITAL. Case wise detailed study was done in all cases by noting the age, sex, social status, nature of violence, duration of the injury and information regarding medical problems and any local problems in relation to bone and joints. The study includes participants with Only Intercondylar fracture humerus. Age more than 18 years. Patient who gave consent for study. Exclusion criteria: Intercondylar fracture humerus associated with diaphysis of humerus or proximal humerus. Intercondylar fracture humerus associated with fractures of olecranon or radial head. Patients with co morbid conditions and who were not fit for surgery.On admission, emergency care was given, with special attention to A (air way), B (breathing), C (circulation). A thorough systematic examination is done to rule out other injuries. They were examined for signs of fractures, deformity and any compromise of distal neurovascular status.

A thorough general examination and local examination was performed. Radiological examination of the part and routine investigations 
were carried out. Patients were taken up for surgery as early as possible in all the cases. Old people with medical problems after thorough work up were taken up for surgery, once the patient is fit for surgery. Pre operatively all patients were immobilised in above elbow plaster of pans slab with elevation of limb. Associated injuries were dealt simultaneously or at a later date depending upon convenience. Every effort was made to operate as early as possible and mobilised as early as possible. Criteria taken are history, clinical and radiological and who had AO type and 13.C fractures are taken up surgery. Patients with external wounds and associated injuries waited till the conditions permit for surgery. No vascular injuries were noted in this series.

The average time between admission and operation was 5 to 6 days. In patients with severe blood loss and in hypovolemic shock, it was corrected with intravenous fluids and blood. For simple fractures antibiotic regimen was started 12 hours before surgery parenterally and continued till fifth postoperative day, from then till $10^{\text {th }}$ postoperative day oral preparation was given. All patients were taken up for surgery when general condition was stable under general anaesthesia or brachial block. The implants used were Recon plates, DCP, 1/3 tubular plates and cancellous screws. Patients were kept in postoperative ward for first 24 hours and then shifted to respective general ward. On $2^{\text {nd }}$ post-operative day drain was removed and antiseptic dressing was done. Postoperative check X-ray was taken. Bed side exercises (active assisted and gravity assisted exercises) were started as early as possible depending on the condition of patient and stability of fracture fixation. Suture removal was done on 10th post-operative day and patient was discharged with advice of active assisted exercises and physiotherapy. Follow up was 6 weekly until time of radiological union.

During the follow up patients were received in outpatient department once in every 3 weeks and fracture union was assessed clinically and radiologically.
For functional evaluation of the results we have choosen Cassebaum's scale.

\section{The Rating System of Cassebaum}

Excellent: Extension deficit of 15 degrees or less and flexion to 130 Degrees or more

Good: Extension deficit of 15 to 30 degrees and flexion of 120-130 degrees

Fair: Extension deficit of 30- 40 degrees and flexion to 90-120 degrees

Poor: Extension deficit of 40 degrees or more and flexion to less than 90 Degrees

\section{Results}

In the past two years starting from July 2015 to September 2017, 20 cases of intercondylar fractures of humerus were treated in the orthopaedic department of NRI medical college. Their analysis is as follows:

Sex Incidence: In this series males sustained more number of fractures than females, as general trauma is more common in males when compared to females.

Table Sex Incidence

\begin{tabular}{|c|c|c|}
\hline Sex & No. Of Cases & Percentage \\
\hline Male & 15 & $75 \%$ \\
\hline Female & 5 & $25 \%$ \\
\hline
\end{tabular}

Age incidence: Most common age group was between 18 to 30 years.

Table Age Incidence

\begin{tabular}{|c|c|c|}
\hline Age & No. Of Cases & Percentage \\
\hline $\mathbf{1 8 - 3 0}$ & 10 & $50 \%$ \\
\hline $\mathbf{3 1 - 4 0}$ & 4 & $20 \%$ \\
\hline $\mathbf{4 1 - 5 0}$ & 4 & $20 \%$ \\
\hline $\mathbf{5 1 - 6 0}$ & 2 & $10 \%$ \\
\hline
\end{tabular}

Table: Side Incidence

\begin{tabular}{|c|c|c|}
\hline Side & No. Of Cases & Percentage \\
\hline Right & 12 & $60 \%$ \\
\hline Left & 8 & $40 \%$ \\
\hline
\end{tabular}

Predisposing factors and etiology

In the present series all the cases had history of trauma either direct or indirect type. Out of the 20 cases 16 were due to RTA and 4 cases were due to fall by various mechanisms. Therefore it is 
apparent that $80 \%$ cases are due to high to moderate velocity injuries and $20 \%$ cases due to moderate to low velocity injuries.

Table: Etiology

\begin{tabular}{|c|c|c|}
\hline Etiology & No. Of Cases & Percentage \\
\hline Rta & 16 & $80 \%$ \\
\hline Fall & 4 & $20 \%$ \\
\hline
\end{tabular}

Type of fracture - incidence - AO classification

The cases taken up for study were classified according to AO classification

AO13.C1: 8 cases, AO13.C2: 8 cases, AO13.C3:

2 cases, AO13.B1: 1 case, AO13.B3: 1 case

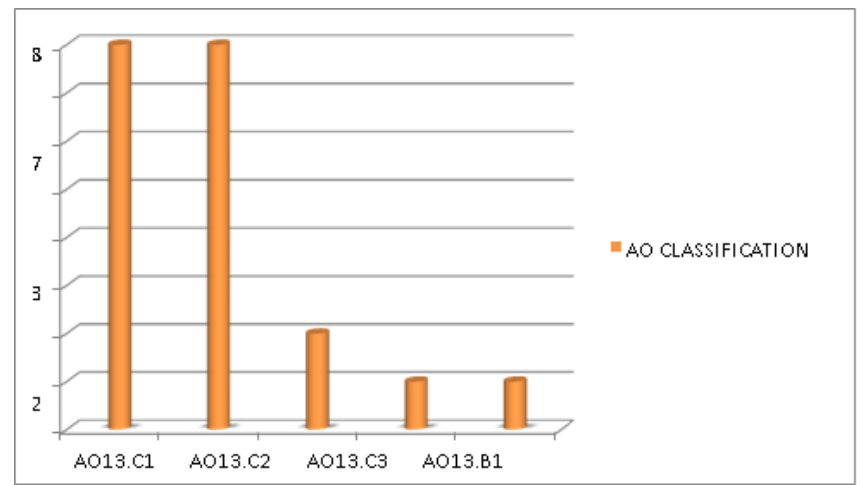

Gustilo-Anderson Classification:

Of the 20 patients taken up for study 2 had open fractures which were classified according to Gustilo-Anderson classification.

Closed Fractures: 18, Grade I Compound: 1, Grade II Compound: 1,

Grade III Compound : 0

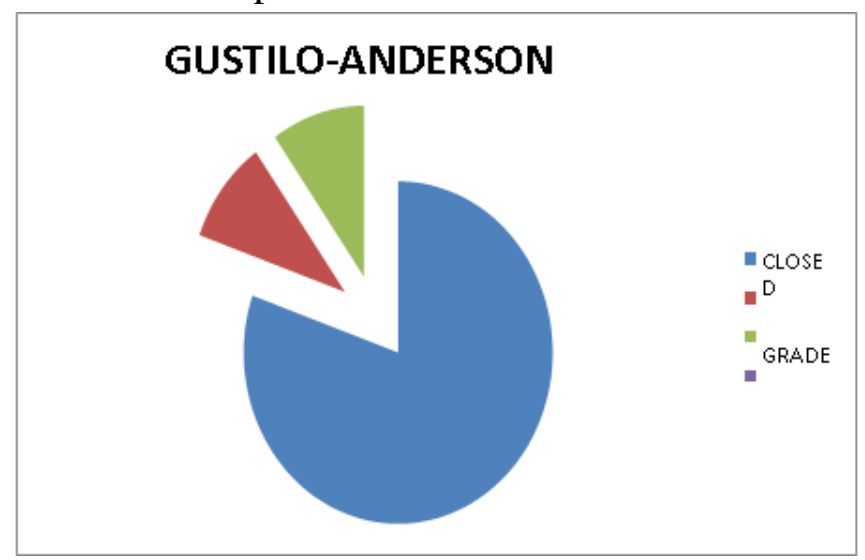

Mode of management:

All the cases were treated by open reduction and internal fixation either with recon plating or locking distal humerus plating or cc screw fixation or External fixation or $\mathrm{K}$ wires. These 20 were taken up for study.

\begin{tabular}{|c|c|}
\hline Mode Of Management & No. Of Cases \\
\hline Recon Plates & 12 \\
\hline $\begin{array}{c}\text { Locking Distal } \\
\text { Humerus Plates }\end{array}$ & 5 \\
\hline Cc Screws & 1 \\
\hline K Wires & 1 \\
\hline External Fixation & 1 \\
\hline
\end{tabular}

Complications

No surgery is without complications even in the best hands. But it is careful selection of cases and good technical skill that decreases the overall bad results. Our patients too had some complications as we found out during our follow-up, which are as follows:
.Infection (superficial)
. Elbow stiffness
. Hardware pain
. Olecranon bursitis

(this is low compared to other studies but the number of cases in our study are less compared to other studies)

Criteria for assessment of results:

The rating system of Cassebaum was adopted to quantitate the final functional motion of the elbow. The data on elbow motion was combined with the patient's subjective symptoms to provide an overall functional rating. An excellent rating was given for a symptom free elbow with a normal or nearly normal range of motion, a good overall rating, for good or excellent elbow motion with some subjective symptoms; a fair rating; for a fair range of motion of the elbow with or without symptoms; and a poor rating for both limited mobility and limited function. 
Cassebaum's Scale: Assessment of results

\begin{tabular}{|c|c|c|c|c|c|}
\hline RATING & MOTION & $\begin{array}{l}\text { RANGE OF } \\
\text { MOTION }\end{array}$ & PAIN & DISABILITY & $\begin{array}{l}\text { NO. OF } \\
\text { CASES } \\
\end{array}$ \\
\hline XCELLENT & \begin{tabular}{|c|} 
Normal \\
or \\
near normal \\
\end{tabular} & $\begin{array}{c}0-15 \text { to } 130 \text { or } \\
\text { more }\end{array}$ & none & none & 10 \\
\hline GOOD & \begin{tabular}{|c|} 
Slight \\
limitation \\
\end{tabular} & $\begin{array}{c}15-30 \text { to } 120- \\
130\end{array}$ & occasional & minimum & 5 \\
\hline FAIR & $\begin{array}{l}\text { Moderate } \\
\text { limitation }\end{array}$ & $\begin{array}{c}30-40 \text { to } 90- \\
120\end{array}$ & $\begin{array}{c}\text { With } \\
\text { activity }\end{array}$ & moderate & 3 \\
\hline POOR & $\begin{array}{c}\text { Marked } \\
\text { limitation }\end{array}$ & $\begin{array}{c}40 \text { or more to } \\
\text { less } \\
\text { han } 90 \text { degrees }\end{array}$ & Variable & severe & 2 \\
\hline
\end{tabular}

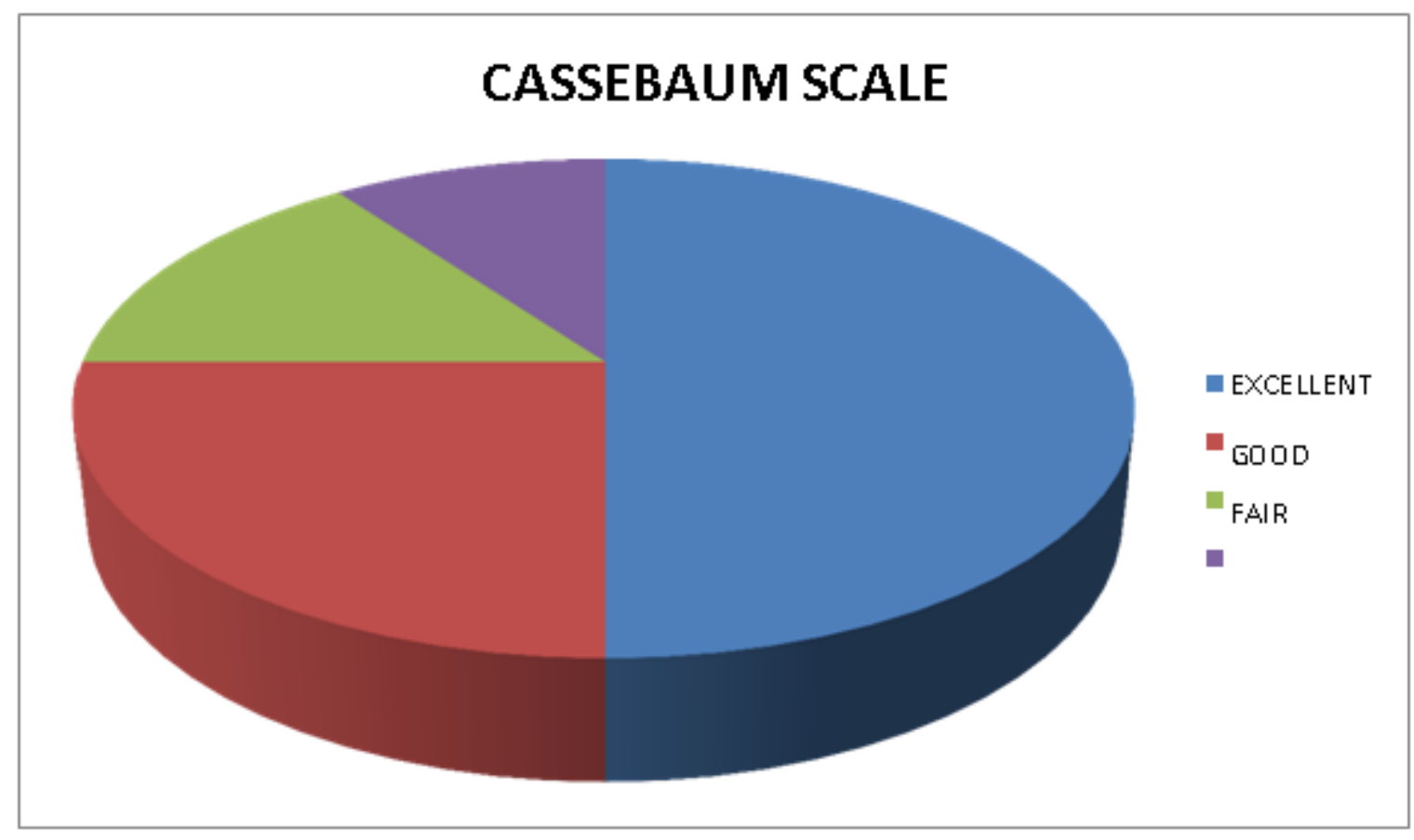

\begin{tabular}{|c|c|c|c|c|c|}
\hline IMPLANT USED & $\begin{array}{c}\text { NUMBER } \\
\text { OF CASES }\end{array}$ & EXCELLENT & GOOD & FAIR & POOR \\
\hline RECON PLATES & 12 & 5 & 4 & 3 & - \\
\hline LOCKING PLATES & 5 & 5 & - & - & - \\
\hline CC SCREWS & 1 & - & 1 & - & - \\
\hline K WIRES & 1 & - & - & - & 1 \\
\hline EXTERNAL FIXATION & 1 & - & - & - & 1 \\
\hline
\end{tabular}

As compared to other modalities of fixation plating proves superior in our study. This coincides with Huang Ji et.al ${ }^{4}$, Ravikant Das et.al ${ }^{5}$ and Hongwei Chen et.al ${ }^{6}$ studies. 


\section{JMSCR VoI||07||Issue ||06||Page 905-915||June}

Case - 1

Pre Op X-Ray

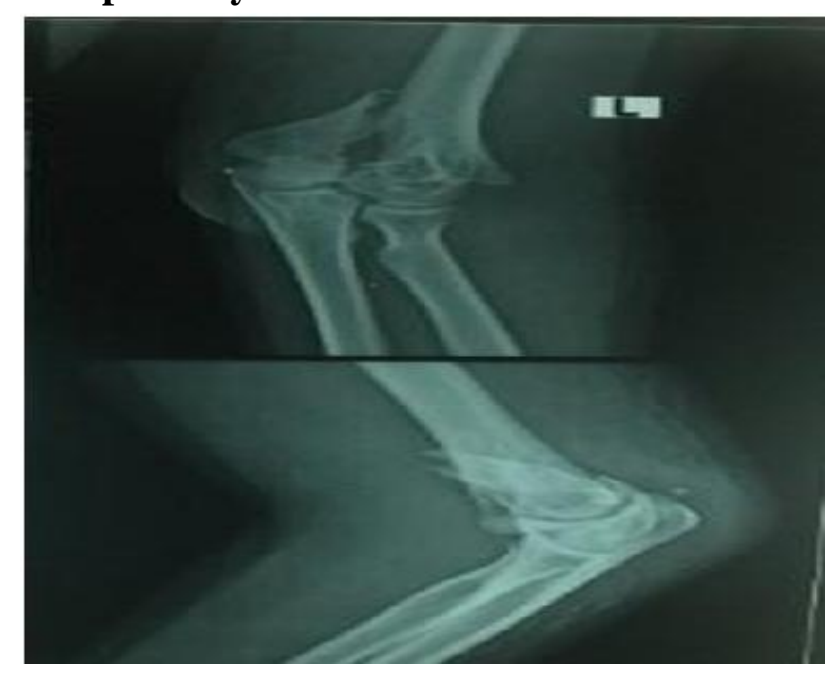

\section{Intra Op}

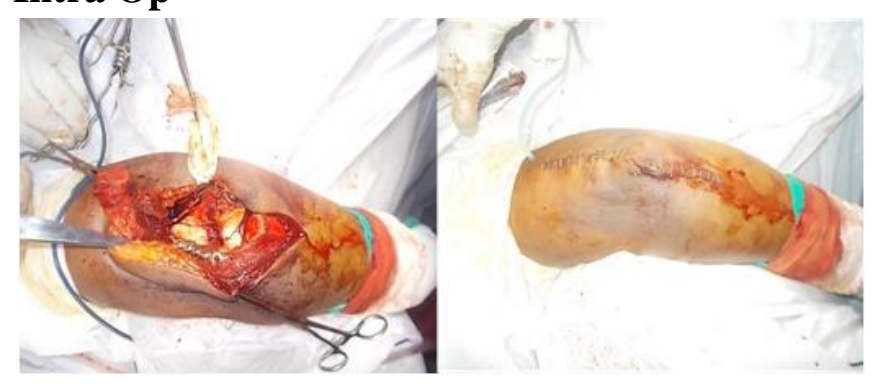

\section{Post Op X-Rays}

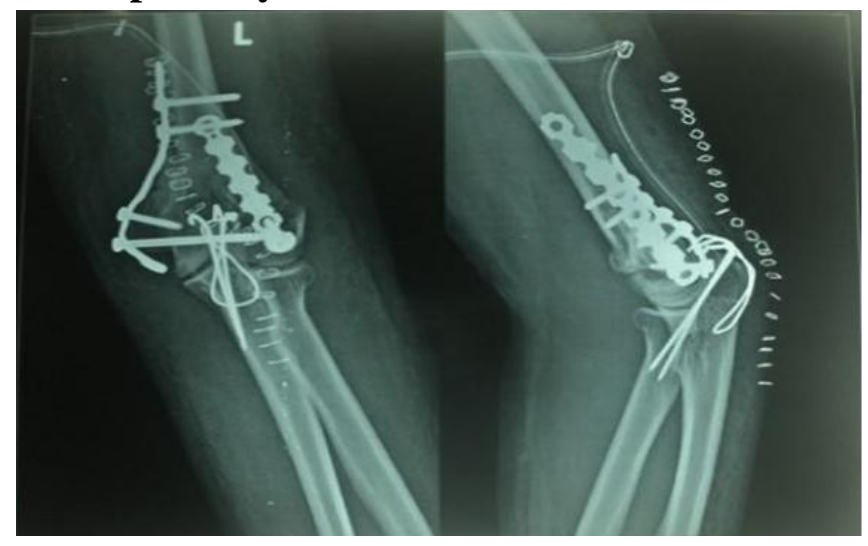

Follow Up

\section{Extension}

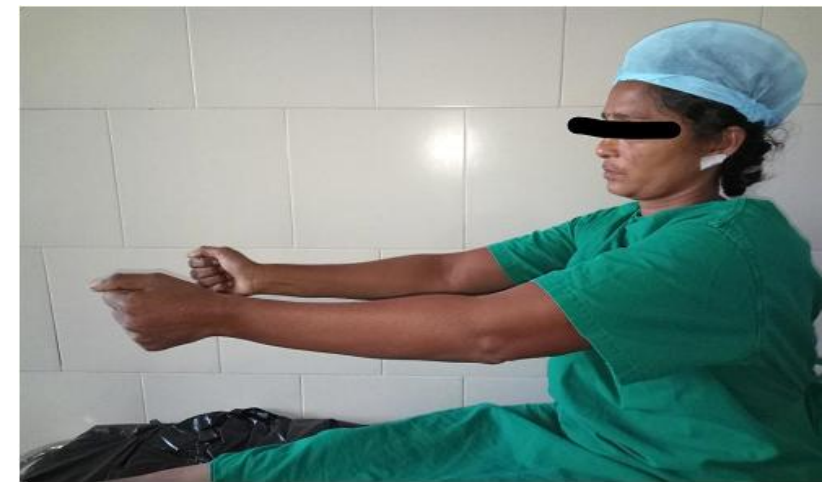

\section{Flexion}

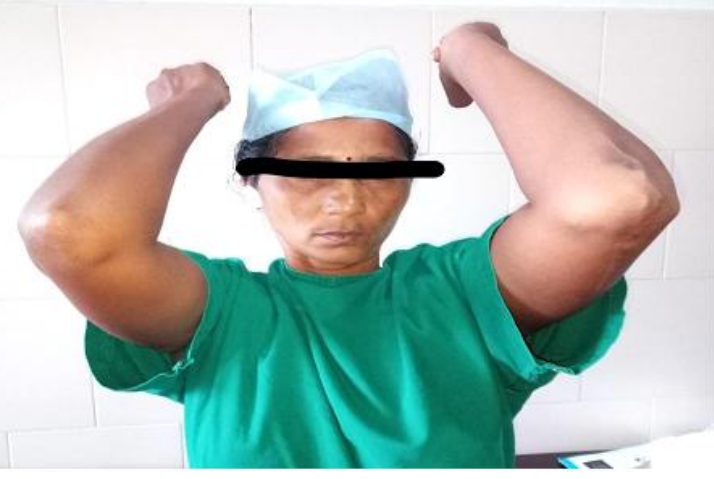

Case - 2

Pre Op X-Ray

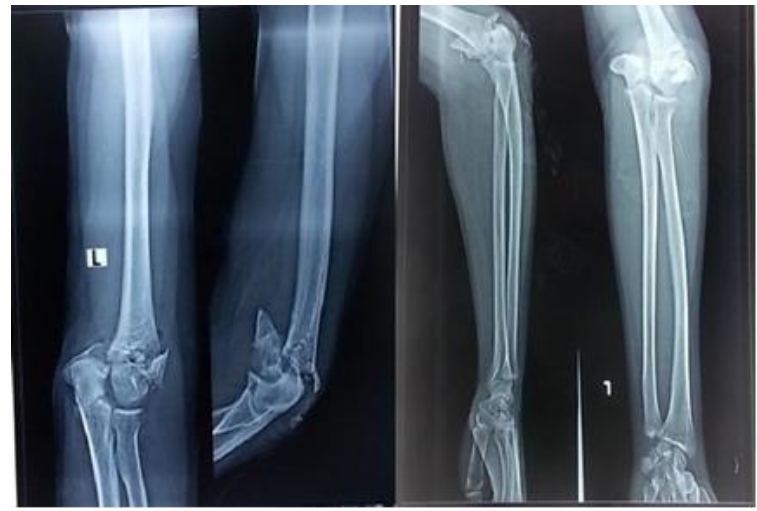

Post Op X-Ray

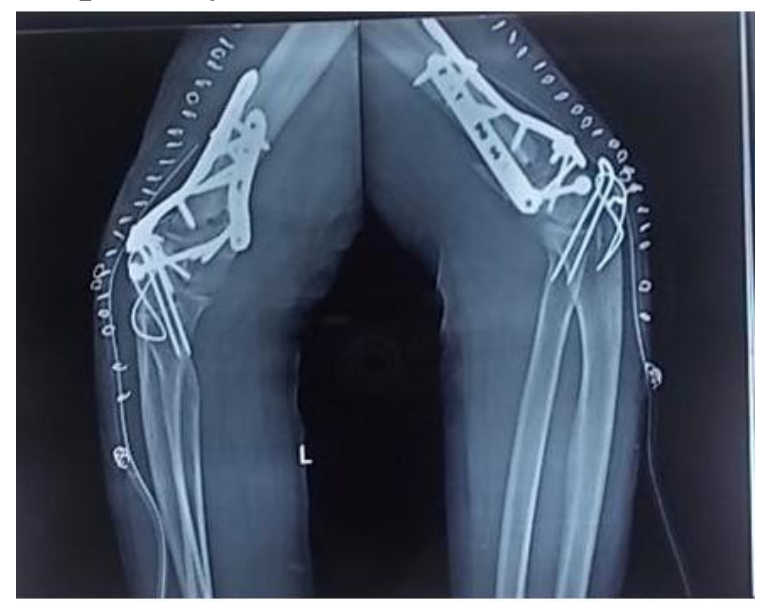

Follow Up

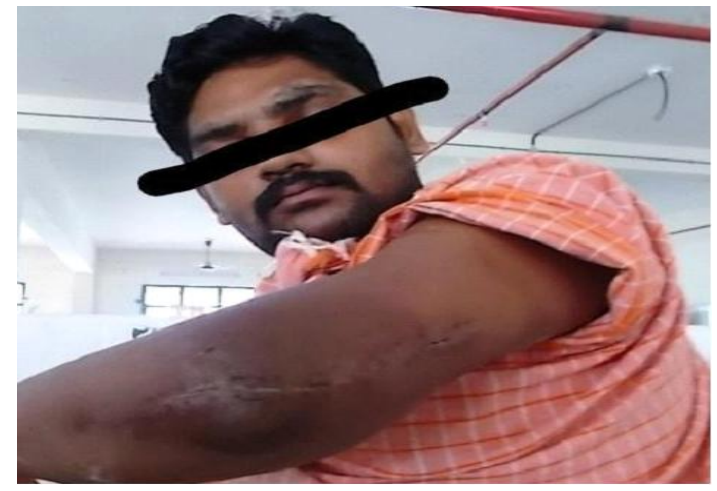




\section{JMSCR VoI||07||Issue||06||Page 905-915||June}

Case - 3

Pre Op X-Ray and CT Scan

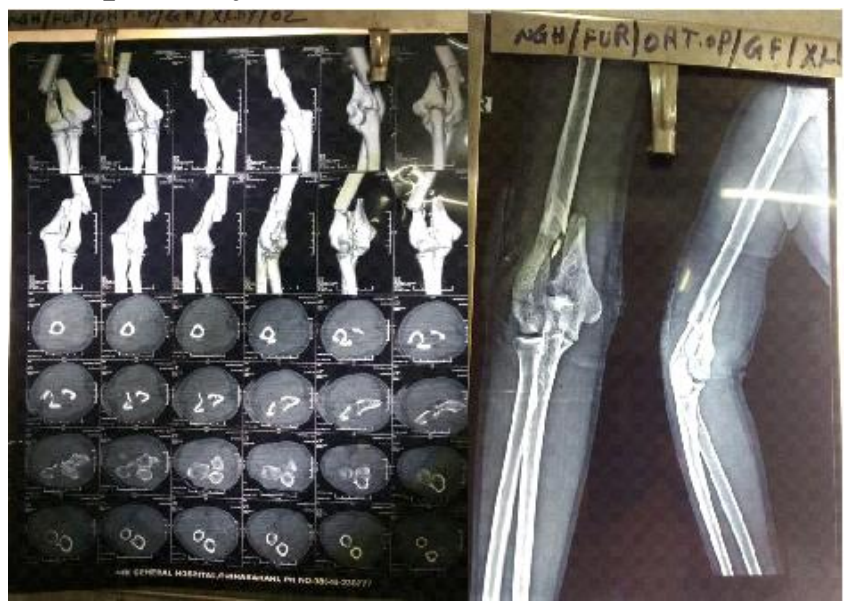

\section{Post Op X-Ray}

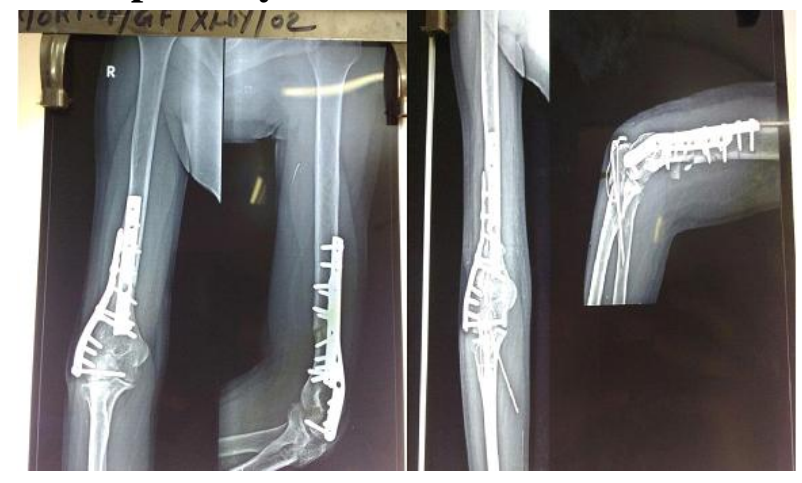

\section{Follow Up}

\section{Flexion}

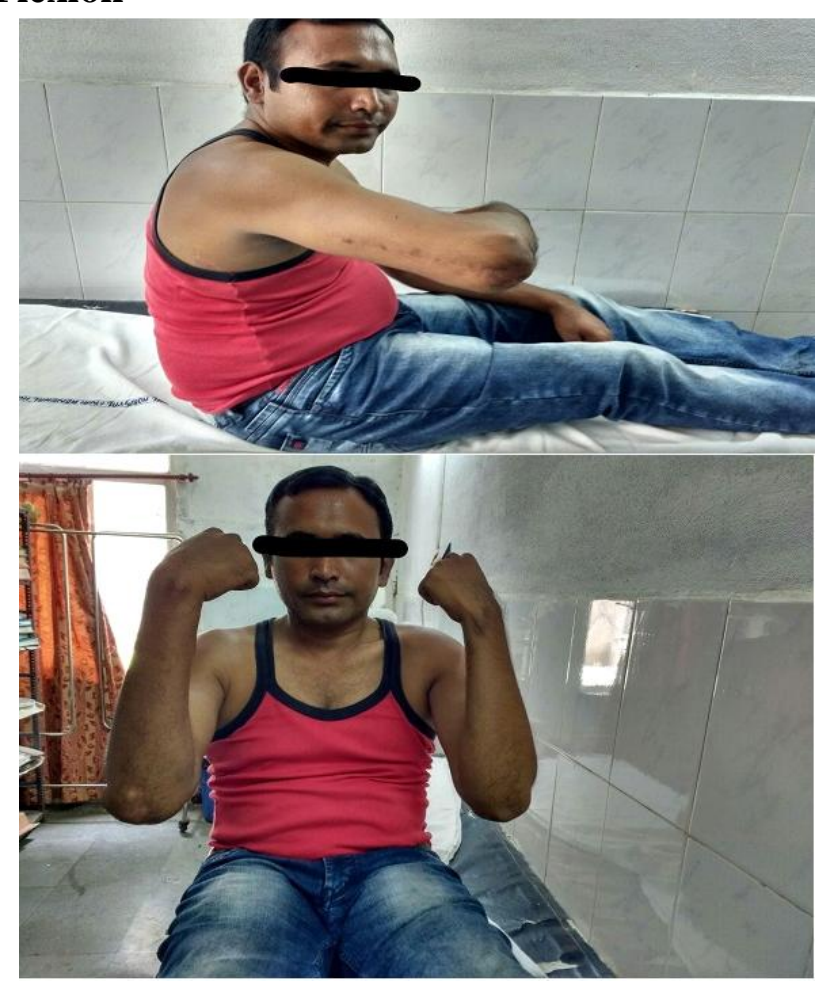

\section{Extension}

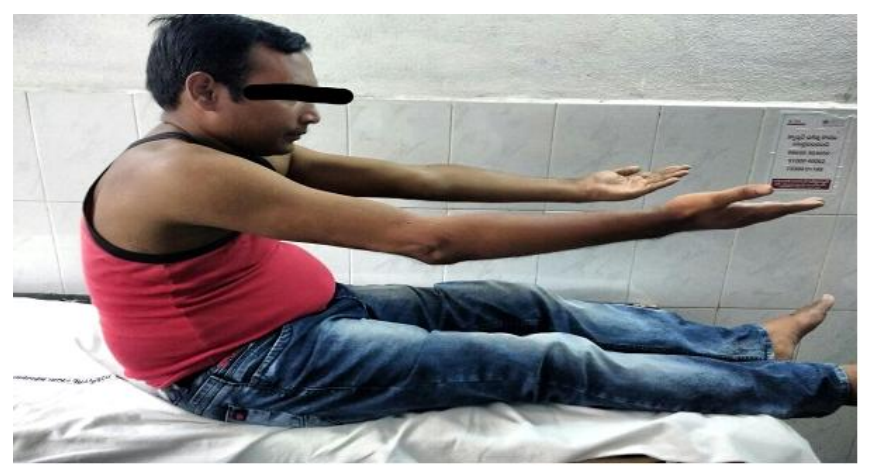

\section{Discussion}

Distal humerus fractures have a bimodal distribution with a broad separation in frequency between younger male patients with high energy mechanisms of injury and older female patients with low energy osteoporotic fractures ${ }^{7}$. Improved longevity in many parts of the world has resulted in an increase in the latter category of fractures ${ }^{8}$. Intercondylar fractures of the distal humerus in adults are difficult fractures to treat because of their rarity and associated significant comminution. The results of managing these fractures non-operatively are limited by failure to get anatomical reduction and early mobilization, which often results in painful stiff elbow and/or pseudarthrosis. Hence an operative management with anatomical reduction of the fragments becomes the treatment of choice for these fractures $^{1}$ and other options include TOTAL ELBOW ARTHROPLASTY [TEA]. However available clinical data are insufficient to permit solid recommendations for the use of $\mathrm{TEA}^{9}$. Fractures of the distal humerus are difficult to treat both by nature of the injury and because most surgeons don't have a great deal of experience with them. The management of intercondylar and comminuted intra-articular distal humerus fractures have been controversial. Advocates of closed treatment felt that operative treatment was technically difficult, fraught with complications and inconsistent results. Advocates of operative management stressed the importance of anatomical restoration of elbow joint for stability and function. While there have been numerous studies regarding the management of this 
uncommon fracture, the overall number of the reported cases has been small, the fracture has been classified by varying criteria, and the results have been judged by wide range of methods of functional evaluation. Diversity has been found even with individual series, as different treatment recommendations were proposed for selected types of fracture patterns. Accurate comparisons between operative and non-operative treatment remained difficult, even within the series of individual authors.

The smaller ASIF screws and plates, cannulated cancellous screws, have added measurably to the versatility of the skeletal fixation, in particular with the comminuted type C3 fractures. Cannulated cancellous screws over $\mathrm{K}$ wires accurately visualises path of the screw, in addition the guide wire maintains the reduction and controls the fracture fragments which is very useful in comminuted intercondylar fracture of distal humerus. The biomechanical evidence in studies of orthogonal and parallel plating for fractures of distal humerus indicates that parallel plating provides greater stiffness in terms of certain test criteria, consisting of axial loading, varus loading, loading in extension, cyclic loading, and load to failure. Theoretically, this makes sense, as both of the plates in a parallel plating construct are aligned with the plane of motion of the elbow. The other advantage of parallel plate configuration is the greater number of screws that it allows to be inserted across the trochlea and capitellum from both the lateral and medial sides of the humerus, providing the benefit of greater screw interdigitation and thus increasing the support provided to the joint surface. This would be of greatest benefit in osteoporotic fractures ant it is in these fractures that parallel plating is most indicated. The two biomechanical models of distal humerus fractures with the best design, used by Got et.al and Stoffel et.al in their respective studies, led to different findings. Got et.al found no difference between orthogonal plating and parallel plating except that orthogonal plating had greater stiffness in torque to failure ${ }^{13}$, where as Stoffel et.al found parallel plating to have greater stiffness under most of the test conditions they applied ${ }^{11}$.

Apart from the study done by Got et.al., none of the studies that used a fracture model with a intercondylar split utilized an interfragmentary screw. However, the use of one or more such screws for achieving interfragmentary compression remains an important part of our surgical plan for treating distal humerus fractures with orthogonal plates, in that the screws effectively restore the joint surface from its fragmented state. Another important realization obtained from the biomechanical studies described above is the clinical relevance of stiffer construct for fracture fixation. Three of these studies observed that although parallel plating was stiffer than orthogonal plating under conditions used in their testing, orthogonal and parallel plating showed no difference in testing under physiologic loads $^{12}$. There is similarly little evidence to support the use of locking screws rather than conventional screws in plate constructs for the fixation of distal humerus fractures. The biomechanical evidence leading to this conclusion resembles that for parallel plating, in that locking screws create a stiffer construct in some but not all testing environments ${ }^{13}$. Clinically, however, Berkes et.al ${ }^{14}$ retrospectively reviewed $96 \mathrm{AO}$ type 13C distal humerus fractures, of which 65 underwent plate fixation with locking screws and 31 with non locking screws, and found no difference in the two groups in rates of non union, infection and re operation. The critical factors for successful outcome remain those advocated earlier by Cassebaum ${ }^{15}$. These include meticulous surgical technique, stable internal skeletal fixation, and early controlled post-operative mobilisation. The careful identification and temporary fixation of the articular fragments with Kirschner wires enables the surgeon to accurately assess the anatomy of individual fracture and to tailor the placement and type of internal fixation to meet each fracture's unique requirements. Concern has been expressed that the extensive dissection and surgical trauma of the exposure required to achieve rigid internal fixation will lead 
to soft tissue fibrosis and limitation of motion. The trans-olecranon approach with the patient in lateral position offers excellent approach of the articular surface and distal end of humerus without the soft tissue trauma, associated with the triceps splitting or tongue of triceps approach. While the trans-olecranon approach requires the creation of an additional intra-articular fracture, this approach also facilitates identification of and protection of ulnar nerve. Anterior transposition is indicated when the nerve is contused from the original trauma or intra-operative retraction or when the metal implants will cause mechanical irritation.

Emphasis was placed on the accurate restoration of the trochlea. The inherent stability provided by its congruent relationship with the greater sigmoid notch of the proximal part of the ulna makes it's anatomical reconstruction important in restoring elbow function and offsetting later degenerative arthritis. In those fractures in which there was loss of trochlear substance due to extensive comminution, autogenous grafts of cancellous bone helped to reconstruct the defect and maintain the overall width of the trochlea. A number of classification systems have been proposed for various patterns found in intercondylar fractures of the distal end of the humerus. The classification system of Muller's et al (AO) is used in this study because it is well suited for operative conditions.

The rating system of Cassebaum has been adopted for use in this study. It is noteworthy that the clinical evaluation did not always correlate with the follow up radiograph. Patient with an excellent overall rating may have radiographic evidence of joint space narrowing or marginal spur formation. Though parallel plating proposed by O'Driscoll $\mathrm{SW}^{16}$ is more biomechanically stable than perpendicular plating technique, we did perpendicular plating technique because it requires less soft tissue dissection and time duration, technically less demanding and stability achieved by this technique is enough for bone union and early rehabilitation. In our study, we treated 14 cases with open reduction and internal fixation with dual plating in 90-90 configuration i.e., one plate on medial border and another on posterior surface of lateral column and 3 cases with open reduction and internal fixation with parallel configuration plating. In the present study the average age was 33.6 years with least age of 21 years and highest age of 58 years. The male/female ratio 15:5 of the 20 cases taken up for study, all were followed up. The study is comparable to the study by Henley et.al ${ }^{17}$ and in Wang et.al ${ }^{18}$ which showed $60 \%$ males and $40 \%$ females. Those cases that did'nt turn up for follow up were not included in the study. In our study, high velocity RTAs were the main cause of most of the fractures involving about 16 cases [80\%] and 4 cases [20\%] were due to direct fall as comparable to Henley et.al study showed $61 \%$ cases due to RTA and $39 \%$ due to direct fall. In our study, the incidence of RIGHT upper limb involvement is 12 cases [60\%] and the incidence of LEFT upper limb involvement is 8 cases [40\%] which is comparable to studies done by Jupiter et.al ${ }^{19}$.

All patients were taken up for surgery under general anaesthesia or regional anaesthesia after being evaluated for their fitness for surgery. Surgical approach was olecranon osteotomy or paratricepital approach. All the fractures were reduced to maintain articular surface and fixed with K. wires, cancellous/cannulated cancellous screws, $3.5 \mathrm{~mm}$ plates along with tension band wire or cancellous screw fixation for olecranon osteotomy. Post-operative period was uneventful in all patients. Early mobilisation started in many cases, except in those cases with severe osteoporosis. Patients were discharged with advice of active, assisted active range of motion exercisers. At follow-up, patients were assessed clinically and radiologically. Patients were followed up for an average of 7 months (range 6 months - 9months), excellent results were found in 10 cases (50\%), Good results in 5 cases $(25 \%)$, fair results were obtained in 3 cases $(15 \%)$ and poor result in 2 cases (10\%). Thus good and excellent results were found in $75 \%$ of cases which is comparable with other studies such as Gupta $\mathrm{R}^{1}$ in 1996 who studied 20 cases of 
intercondylar fracture humerus treated with open reduction and internal fixation and early mobilization and achieved good and excellent results in 15 cases $(75 \%)$. The inclusion criteria being similar. Another study by Allende CA et $\mathrm{al}^{20}$ in 2004 also showed comparable results in which 40 cases were studied which were surgically treated and the inclusion criteria was similar showed good and excellent results in 34 cases $(85 \%)$. The two patients with poor results in our study, one had an open fracture (grade II) and the fixation was done with External fixation. $\mathrm{He}$ had postoperative complication of superficial infection and elbow stiffness.

The other patient had an open fracture (grade I) and the fixation was done with $\mathrm{K}$ wires. $\mathrm{He}$ had post operative complication of superficial infection. All these factors were responsible for the poor result. Hence it is important to fix the fracture once the infection subsides. No postoperative permanent nerve palsies were found. Hardware pain was noted in 2 patients. They were pain free after removal of the implant. Until the fracture was united for the implant removal these patients were prescribed mild analgesics. In summary, the concept of open reduction and internal fixation of intercondylar fractures of the humerus with dual plates is very valuable, in restoring articular surface and early rehabilitation decreasing morbidity, resulting good results.

\section{Conclusion}

In surgical management of intercodylar fractures of the distal humerus, anatomical reduction and rigid internal fixation is atmost important, as well as to accurately reconstruct the articular surface. Early post operative mobilisation by active assisted exercises and physiotherapy is must for good functional outcome. Poor fixation requiring prolonged post-operative immobilisation however will invariably produce unsatisfactory functional results. For open reduction and internal fixation of intercondylar fractures of humerus posterior approach with olecranon osteotomy is the best. No other approach known to expose the elbow joint, allows so great a freedom of selection of fixation materials, does so little harm to important anatomical structures, or offers such an excellent view of the articulating surfaces. 20 cases of intercondylar fractures of humerus have been done by open reduction and internal fixation, 10 cases had excellent results, 5 had good, 3 had fair and 2 had poor results in our study. Hence dual plating, prevention of infection, early postoperative mobilisation and physiotherapy are essential for excellent results. In young, the results are better than in older patients probably because of active cooperation in physiotherapy by young and the quality of good bone stock compared to elderly patients.

\section{References}

1. Gupta R- Intercondylar fractures of distal humerus in adults. Injury. 1996 Oct;27(8):569-72.

2. Rockwood \& Green's Fractures in Adults, 6th Edition Copyright A@2006 Lippincott Williams \& Wilkins

3. Jupiter JB, Morrey BF. Fractures of the distal humerus in the adult: The elbow and its disorders, 2nd ed. PhiIadelphia: wB Saunders, 1993:328a€"366.

4. Huang Jl, Paczas M, Hoyen HA, Vallier HA. Functional outcome after open reduction internal fixation of intra-articular fractures of the distal humerus in the elderly. J Orthop Trauma. 2011 May;25(5):259-65.

5. Ravikant Das, Arunesh Singh, Pranay Shrivastava, Atul Manoharrao Deshkar. "Long Term Outcome of Surgical Management of Intercondylar Fracture of Humerus". Journal of Evolution of Medical and Dental Sciences 2015; Vol. 4, Issue 40, May 18; 6954-6959, DOI: 10.

6. Hongwei Chen, Dacheng Li, Jun Zhang \& Xinwei Xiong: Comparison of treatments in patients with distal humerus intercondylar fracture: a systematic review and meta-analysis 613-625 | 2017, Published online: 14 Jun 2017

7. Robinson CM, Hill RM, Jacobs N, Dall G, 
Court-Brown CM. Adult distal humeral metaphyseal fractures: Epidemiology and results of treatment.J Orthop Trauma 2003;17(1):38-47.

8. Palvanen M, Kannus P, Neimi S, Parkkari J. Secular trends in distal humeral fractures of elderly women: Nationwide statistics in Finland between 1970 and 2007. Bone 2010;46(5):1355-1358.

9. Gay DM, Lyman S, Do H, Hotchkiss RN, Marx RG, Daluiski A. Indications and reoperation rates for total elbow arthroplasty: An analysis of trends in New York state. J Bone Joint Surg Am 2012;94(2): 110-17.

10. Got C, Shuck J, Biercevicz A. Biomechanical comparision of parallel versus 90-90 plating of bicolumn distal humerus fractures with intra articular comminution. J Hand Surg Am 2012;37(12): 2512-2518.

11. Stoffel K, Cunneen S, Morgan R, Nicholls R, Stachowiak G. Comparative stability of perpendicular versus parallel doublelocking plating systems in osteoporotic comminuted distal humerus fractures. J Orthop Res2008;20(1):12-20.

12. Sabalic S, Kodvanj J, Pavic A. Comparative study of three models of extra articular distal humerus fracture osteosynthesis using the finite element method on an osteoporotic computational model.Injury 2013;44(suppl 3):S56-S61. 80

13. Caravaggi $P$, Laratta JL, Yoon RS. Internal fixation of the distal humerus: A comprehensive biomechanical study evaluating current fixation techniques. J Orthop Trauma 2014;28(4):222-226.

14. Berkes M, Garrigues G, Solic J. Locking and non-locking constructs achieve similar radiographic and clinical outcomes for internal fixation of intra-articular distal humerus fractures. Hssj 2011;7(3): 244-50.
15. Cassebaum WH :Operative treatment of $\mathrm{T}$ and Y Fractures of lower end of humerus, Am J Surg. 1952 Mar;83(3):265-70

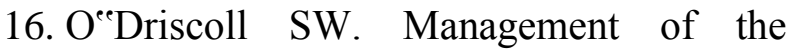
smashed distal humerus. Orthop Clin N Amer. 2002;33:19-33.

17. 49. Henley MB; Intraarticular distal humerus fractures in adults. Orthop clin north Am.,1987;18(1): 11-23.

18. 50. Wang KC, Shih HN, Hsu KY, Shih $\mathrm{CH}$. Intercondylar fractures of the distal humerus: Routine anterior subcutaneous transposition of the ulnar nerve in a posterior operative approach. J Trauma. 1994;36(6):770-3.

19. Jupiter JB, Neff U, Holzach P. Intercondylar fractures of the humerus: an operative approach. J Bone Joint Surg Am. 1985;67(2):226-38.

20. Allende CA, Allende BT, Allende BL, Bitar I, Gonzalez G. Intercondylar distal humerus fractures-surgical treatment and results. Chir Main. 2004 Apr;23(2):85-95. 